\section{ORIGINAL} RESEARCH

\section{J.L. Chazen}

A. Gupta

A. Dunning

C.D. Phillips

\title{
Diagnostic Accuracy of 4D-CT for Parathyroid Adenomas and Hyperplasia
}

BACKGROUND AND PURPOSE: 4D-CT is a novel method of multiphase CT imaging. When used to localize parathyroid adenomas and hyperplasia, this technique may allow for more robust diagnostic accuracy than traditional sonography and nuclear scintigraphy techniques. The purpose of our study is to assess the accuracy of 4D-CT for localizing pathologically proved parathyroid adenomas and hyperplasia found during surgery.

MATERIALS AND METHODS: A total of 35 pathologically proved cases of parathyroid adenoma and hyperplasia were retrospectively reviewed between January 2009 and March 2011. Inclusion criteria were availability of final surgical pathology and performance of preoperative 4D-CT. No cases were excluded. Sensitivity, specificity, and accuracy of 4D-CT were ascertained including both the side and quadrant of the pathologically proved lesion.

RESULTS: Of the 35 pathologically proved cases collected over the study period, 32 (sensitivity = $91 \%$ ) patients were found positive for parathyroid disease using 4D-CT, including 3 cases of multigland disease. For lateralization of single-gland disease, 4D-CT demonstrated an accuracy of 93\%. 4D-CT revealed a suboptimal $44 \%$ sensitivity, but $100 \%$ specificity, for multigland disease.

concLusions: 4D-CT demonstrated a high diagnostic accuracy for single and multigland disease in our cohort. Importantly, 4D-CT accurately lateralized single-gland adenomas in $>90 \%$ of cases, allowing the surgeon to employ a directed operative approach. 4D-CT also showed a very high specificity for the detection of multigland disease.

ABBREVIATIONS: $\mathrm{CTDI}=\mathrm{CT}$ dose index; NPV $=$ negative predictive value; $\mathrm{PPV}=$ positive predictive value; $\mathrm{PTH}=$ parathyroid hormone

D irected parathyroidectomy is a minimally invasive surgical technique for parathyroid adenoma resection. When combined with intraoperative parathyroid hormone assay, this technique demonstrates high cure rates, low complications rates, and avoids both general anesthesia and overnight hospital stays associated with classic bilateral neck exploration. ${ }^{1}$ Critical to the success of a directed technique is preoperative localization of the abnormal parathyroid gland. ${ }^{2}$ Traditional imaging modalities such as sonography and sestamibi-SPECT demonstrate high specificity when concordant, but suboptimal sensitivity in most hands. ${ }^{3}$ In addition, ectopic parathyroid adenomas, sometimes located in sonographically inaccessible regions such as the mediastinum, benefit from evaluation with a cross-sectional imaging technique. ${ }^{4}$

The purpose of our study is to assess the accuracy of 4D-CT for localizing pathologically proven parathyroid adenomas and hyperplasia found at surgery. 4D-CT is a novel method of multiphase CT imaging. ${ }^{5-8}$ This technique utilizes the principles of altered arterial enhancement and washout that guided early angiographic detection of parathyroid adenomas. ${ }^{9,10}$ Our hypothesis is that preoperative 4D-CT can accurately localize the abnormal parathyroid gland found at surgery. $4 \mathrm{D}-\mathrm{CT}$ may allow for more robust diagnostic accuracy than

Received January 25, 2011; accepted after revision June 29.

From the Section of Head and Neck Imaging in the Department of Radiology (J.L.C., A.G., C.D.P.) and Division of Biostatistics and Epidemiology in the Department of Public Health (A.D.), Weill Cornell Medical College, New York, New York.

Please address correspondence to C. Douglas Phillips, MD, FACR, Weill Cornell Medical College, New York Presbyterian Hospital, 565 E. 68th St, Department of Radiology, New York, NY 10065; e-mail: cdp2001@med.cornell.edu

http://dx.doi.org/10.3174/ajnr.A2805 traditional sonography and nuclear scintigraphy techniques. ${ }^{5}$ Furthermore, 4D-CT facilitates the detection of coexistent thyroid and mediastinal pathology, and may provide superior accuracy in patients with prior neck surgery. 7,8

\section{Materials and Methods}

\section{Patients}

A waiver of informed of consent was obtained from our institutional review board and was performed in a fashion consistent with the Health Insurance Portability and Accountability Act. We began performing 4D-CT studies at our institution in January 2009 and reviewed all such studies performed at our institution between January 2009 and March 2011 for clinically suspected parathyroid hyperplasia. Inclusion criterion was availability of final surgical pathology. In cases of single-gland disease, a pathologic diagnosis of "parathyroid hyperplasia" was presumed to represent a parathyroid adenoma. This is because, while a specimen may have morphologic features of a parathyroid adenoma, the pathologist cannot exclude the possibility of hyperplasia if only 1 gland was submitted for evaluation. No cases were excluded. Patient demographic data, including age, sex, BMI, and preoperative and postoperative PTH levels, were obtained. For purposes of precise gland localization, lesions were categorized into 4 quadrants.

\section{CT Technique}

All examinations were performed with a LightSpeed 16- or 64-detector row CT scanner (GE Healthcare, Milwaukee, Wisconsin) with a rotation time of $600 \mathrm{~ms}$, scan field of view of head, display field of view of $18 \mathrm{~cm}$, pitch of $0.984: 1$, tube voltage of $120 \mathrm{kVp}$, and automatic exposure control (Smart $\mathrm{mA}$ algorithm, GE Healthcare) with a range from 150-300 mA and noise index of 14 at $1.25-\mathrm{mm}$ section thick- 
ness. Imaging extended from the carina to the bottom of the mandibular teeth in a caudal to cranial direction, in a plane parallel to the hard palate. Each examination included a scout scanogram, noncontrast acquisition, and multiphase imaging following the administration of intravenous contrast.

Iohexol 300 (Omnipaque; GE Healthcare) nonionic iodinated contrast was administered at a dose of $2 \mathrm{~mL}$ per $\mathrm{kg}$ patient weight to a maximum total of $120 \mathrm{~mL}$. Intravenous infusion rate was $4 \mathrm{~mL}$ per second via an 18-gauge catheter.

The diagnostic multiphase CT was acquired at $1.25-\mathrm{mm}$ section thickness. The helical postcontrast sequence was obtained 25 seconds after the start of intravenous contrast administration. Following a subsequent 30 -second delay, a delayed phase sequence was acquired. Axial, sagittal, and coronal 2.5-mm reformations were generated.

\section{Image Analysis}

Images were reviewed prospectively on a PACS workstation by a single attending neuroradiologist with over 20 years of experience in head and neck imaging, and a certificate of added qualification in neuroradiology. Biochemical information was available at the time of interpretation, including the preoperative PTH levels. Images were reviewed and determined to be positive if a soft tissue lesion in the expected location of parathyroid tissue (orthotopic or ectopic) demonstrated early arterial enhancement and qualitatively appreciable contrast washout on early or late delayed images. The radiology report provided a descriptive location for our referring surgeons, with anatomic landmarks. Any coexistent pathology that was deemed to potentially alter patient management was also noted.

\section{Statistical Analysis}

SAS version 9.2 (SAS Institute, Cary, North Carolina) was used for all statistical analysis. Categoric variables are presented as frequencies and percents, while continuous variables are presented as means and standard deviations. To determine how well $4 \mathrm{D}-\mathrm{CT}$ performs in preoperative localization, we used diagnostic accuracy measures comparing 4D-CT to the "gold standard" of surgical pathology. Correct localization of the lesions was ascertained by using side and quadrant of the neck. Diagnostic accuracy measures for both side and quadrant location were determined by using sensitivity, specificity, PPV, NPV, and accuracy, with corresponding 95\% confidence intervals calculated by using the exact binomial distribution method.

\section{Results}

All studies were performed in patients with elevated PTH and a clinical diagnosis of primary hyperparathyroidism. A total of 42 4D-CT studies were performed over the study period. Of these, 35 patients went to surgery with final pathology available and were included in the analysis. The remaining 7 patients were lost to follow-up or refused surgery. No cases were negative for parathyroid hyperplasia on surgical exploration. Table 1 displays the descriptive statistics for our cohort. Of the 35 cases, 32 cases of parathyroid adenoma or multigland hyperplasia were detected by using $4 \mathrm{D}-\mathrm{CT}$ (sensitivity $=91 \%$, 95\% CI 77\%, 98\%). To calculate diagnostic accuracy of 4D-CT to correctly determine side and quadrant location of the lesions, we looked at the 32 cases that were positive by both pathology and 4D-CT. Of these 32 cases, 5 cases were removed because multiple glands were involved. The final single-gland dataset contained 27 cases. Table 2 displays the distribution of the pathology and 4D-CT findings from these 27 individuals.

\begin{tabular}{lcc}
\hline Table 1: Descriptives & & \\
\hline Variable & Mean/Freq & SD/Percent \\
\hline Age $(\mathrm{yr})$ & 59.3 & 9.9 \\
Female gender $(n)$ & 26 & $74 \%$ \\
BMl $\left(\mathrm{kg} / \mathrm{m}^{2}\right)$ & 26.8 & 5.6 \\
Weight $(\mathrm{kg})$ & 73.2 & 16.9 \\
Lesion weight $(\mathrm{mg})$ & 566.7 & 611.0 \\
Prior neck surgery $(n)$ & 2 & $6 \%$ \\
Single-gland disease $(n)$ & 28 & $80 \%$ \\
Multigland disease $(n)$ & 7 & $20 \%$ \\
Peak preoperative parathyroid hormone $(\mathrm{pg} / \mathrm{mL})$ & 190.3 & 288.6 \\
Postoperative parathyroid hormone $(\mathrm{pg} / \mathrm{mL})$ & 29.5 & 27.0 \\
\hline Note:-Freq indicates frequency & &
\end{tabular}

Note:-Freq indicates frequency.

\begin{tabular}{|c|c|c|c|c|}
\hline \multirow[b]{2}{*}{ Variable } & \multicolumn{2}{|c|}{ Pathology Findings } & \multicolumn{2}{|c|}{ 4D-CT Findings } \\
\hline & Mean/Freq & SD/Percent & Mean/Freq & SD/Percent \\
\hline \multicolumn{5}{|l|}{ Lesion detection } \\
\hline Positive & 27 & $100.0 \%$ & 27 & $100.0 \%$ \\
\hline Negative & 0 & $0.0 \%$ & 0 & $0.0 \%$ \\
\hline \multicolumn{5}{|l|}{ Lesion location } \\
\hline Right superior & 6 & $22.2 \%$ & 4 & $14.8 \%$ \\
\hline Right inferior & 6 & $22.2 \%$ & 8 & $29.6 \%$ \\
\hline Left superior & 9 & $33.3 \%$ & 8 & $29.6 \%$ \\
\hline Left inferior & 6 & $22.2 \%$ & 7 & $25.9 \%$ \\
\hline \multicolumn{5}{|l|}{ Lesion side } \\
\hline Right & 12 & $44.4 \%$ & 12 & $44.4 \%$ \\
\hline Left & 15 & $55.6 \%$ & 15 & $55.6 \%$ \\
\hline \multicolumn{5}{|l|}{ Lesion z-axis } \\
\hline Superior & 15 & $55.6 \%$ & 12 & $44.4 \%$ \\
\hline Inferior & 12 & $44.4 \%$ & 15 & $55.6 \%$ \\
\hline
\end{tabular}

Note:-Freq indicates frequency.

Fig 1 demonstrates the classic enhancement pattern seen with a parathyroid adenoma. Figs 2 and 3 demonstrate ectopic adenomas detected by using 4D-CT.

We then determined the diagnostic accuracy of 4D-CT to correctly lateralize the adenoma found at surgery. For diagnostic accuracy, pathology was treated as the gold standard. $4 \mathrm{D}-\mathrm{CT}$ test results were correlated to pathology, and each CT result was evaluated and classified as true-positive, true-negative, false-positive, or false-negative in terms of the ability to accurately predict laterality (right versus left). It is important to note that false-positives and false-negatives were equally weighted. The sensitivity of $4 \mathrm{D}-\mathrm{CT}$ to correctly identify a right-sided adenoma was $92 \%(61.5 \%, 99.8 \%)$, and the specificity of $4 \mathrm{D}$-CT to correctly identify a left-sided adenoma was $93.3 \%(68.1 \%, 99.8 \%)$. The overall accuracy of 4D-CT to correctly identify adenoma side (assuming $4 \mathrm{D}-\mathrm{CT}$ was positive) was $93 \%(76 \%, 99 \%)$. Table 3 presents all diagnostic accuracy measures for $4 \mathrm{D}-\mathrm{CT}$ lateralization.

We also evaluated the ability of 4D-CT to precisely localize an adenoma. For this analysis, we looked at the 25 patients with single-gland disease that was correctly lateralized by $4 \mathrm{D}$ CT. Again, pathology was treated as the criterion standard, and $4 \mathrm{D}-\mathrm{CT}$ results were classified as true-positive, true-negative, false-positive, or false-negative in terms of the ability to accurately predict location (superior versus inferior). As before, false-positives and false-negatives are equally weighted. The sensitivity of $4 \mathrm{D}-\mathrm{CT}$ to correctly determine superior adenoma quadrant location was $85.7 \%$ (57.2\%, 98.2\%), and the specificity to correctly determine inferior adenoma quadrant 

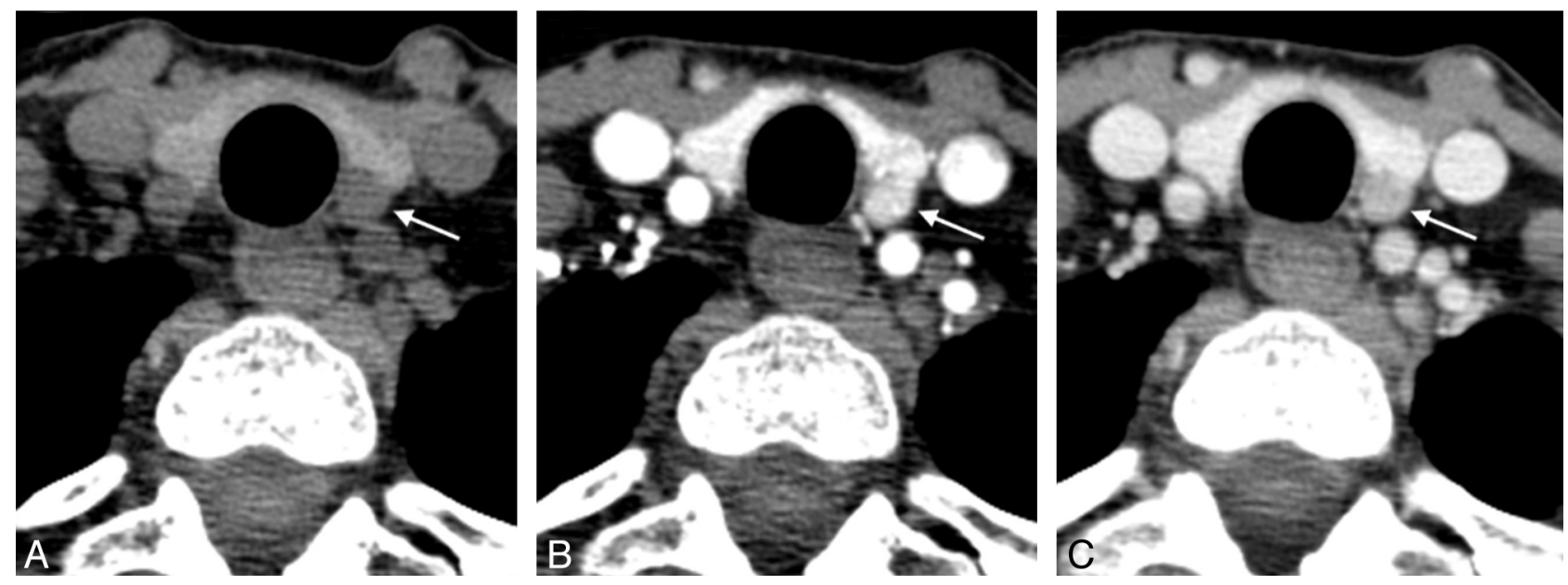

Fig 1. Axial noncontrast $(A)$, axial early phase postcontrast $(B)$, and axial delayed phase postcontrast $(C)$ images show a hypoattenuated hypodense nodule contiguous with the left posterior thyroid gland, which demonstrates avid early contrast enhancement and washout. Pathology revealed a 600-mg parathyroid adenoma.
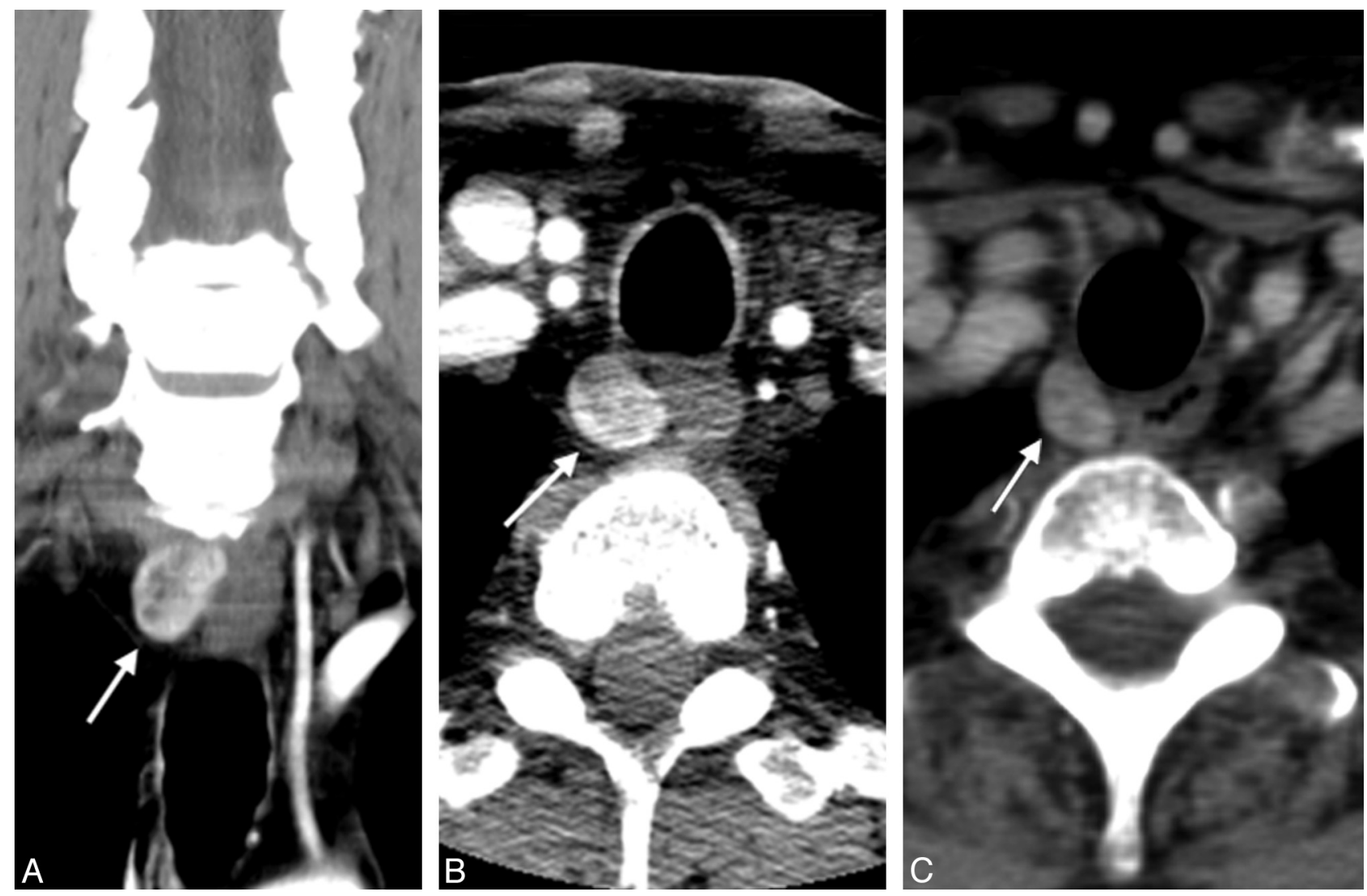

Fig 2. Coronal early phase postcontrast $(A)$, axial early phase postcontrast $(B)$, and axial delayed phase postcontrast $(C)$ images showing an early enhancing 1.3 -cm nodule in the right tracheoesophageal groove, with modest contrast washout representing a right inferior parathyroid gland in an orthotopic location. Pathology demonstrated a 3-g parathyroid adenoma.

location was $100 \%(71.5 \%, 100 \%)$. The overall accuracy of $4 \mathrm{D}-\mathrm{CT}$ to correctly identify precise adenoma location (assuming 4D-CT was positive for adenoma and correctly identified side) was $92 \%(74 \%, 99 \%)$. Table 4 provides all diagnostic accuracy for $4 \mathrm{D}-\mathrm{CT}$ quadrant localization.

Last, we looked at patients with multigland disease (including 4-gland hyperplasia and double adenomas). By pathology, 7 patients in total were determined to have multigland involvement. Of these, 2 (29\%) were called negative for any parathyroid lesion on 4D-CT. We looked at diagnostic accu- racy for multigland disease, both including and excluding patients called negative by $4 \mathrm{D}-\mathrm{CT}$. As before, pathology was treated as the criterion standard, with true-positives defined as those that CT correctly called lesions at multiple locations, false-positives as those that CT incorrectly called not multigland (but are truly multigland), true-negatives as those that CT correctly called not multigland, and false-negatives as those that CT incorrectly called multigland (but are truly not multigland). Looking at all 35 patients, $4 \mathrm{D}$-CT has a low sensitivity of $42.9 \%(9.9 \%, 81.6 \%)$ in detecting multigland dis- 

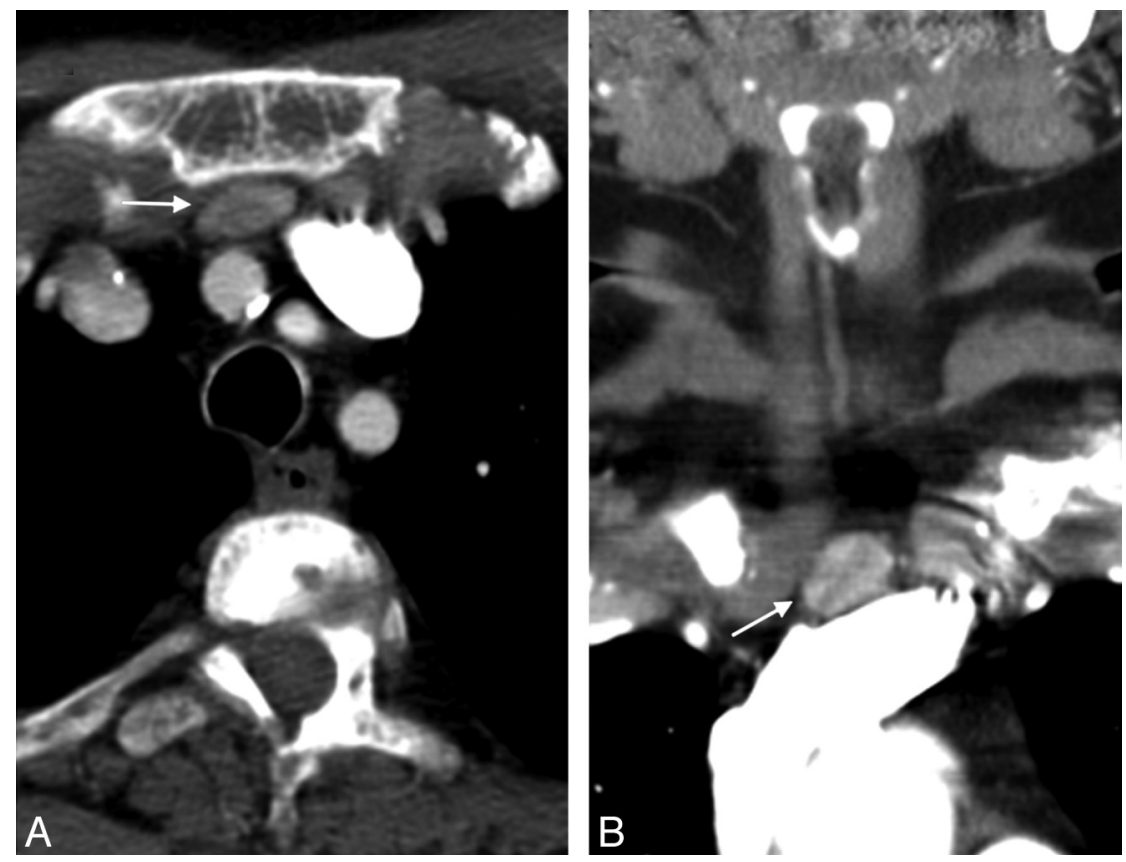

Fig 3. Axial $(A)$ and coronal $(B)$ postcontrast images showing an enhancing retrosternal ectopic parathyroid adenoma.

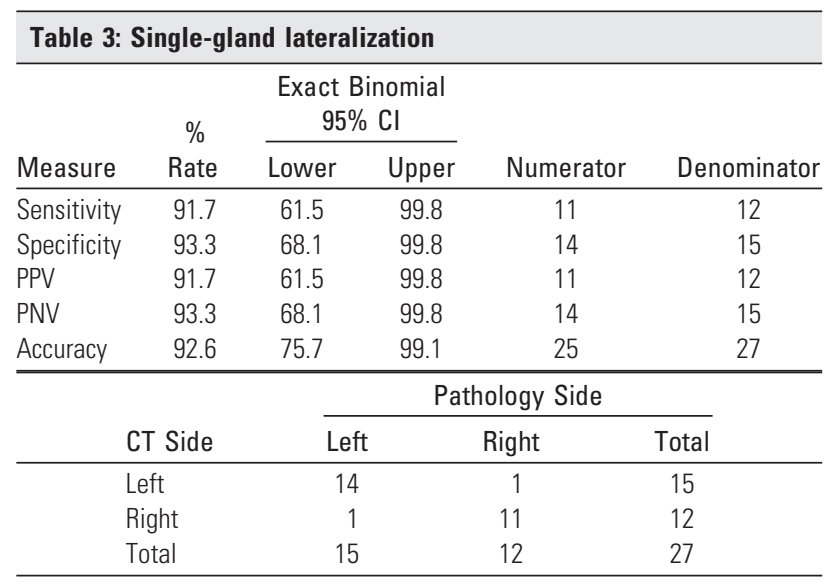

\begin{tabular}{lccccc}
\hline \multicolumn{5}{l}{ Table 4: Single-gland quadrant localization } \\
\hline \multicolumn{5}{c}{ Exact Binomial } \\
\cline { 3 - 5 } Measure & Rate & Lower & Upper & Numerator & Denominator \\
\cline { 3 - 6 } Sensitivity & 85.7 & 57.2 & 98.2 & 12 & 14 \\
Specificity & 100 & 71.5 & 100 & 11 & 11 \\
PPV & 100 & 73.5 & 100 & 12 & 12 \\
PNV & 84.6 & 54.6 & 98.1 & 11 & 13 \\
Accuracy & 92.0 & 74.0 & 99.0 & 23 & 25 \\
\hline
\end{tabular}

\begin{tabular}{lccc}
\hline & \multicolumn{3}{c}{ Pathology Quadrant } \\
\cline { 2 - 4 } CT Quadrant & Inferior & Superior & Total \\
\hline Inferior & 11 & 2 & 13 \\
Superior & 0 & 12 & 12 \\
Total & 11 & 14 & 25 \\
\hline
\end{tabular}

ease. However specificity to rule out a multigland disease in this population was perfect (specificity $=100 \%, 95 \% \mathrm{CI}$, $87.7 \%, 100 \%)$. Looking at the 32 patients in whom both pathology and 4D-CT were positive, 4D-CT had a slightly higher sensitivity of $60 \%$ (CI, $14.7 \%, 94.7 \%)$ for detecting multigland

\begin{tabular}{|c|c|c|c|c|c|}
\hline \multirow[b]{2}{*}{ Measure } & \multirow{2}{*}{$\begin{array}{c}\% \\
\text { Rate }\end{array}$} & \multicolumn{2}{|c|}{$\begin{array}{l}\text { Exact Binomial } \\
\qquad 95 \% \mathrm{Cl}\end{array}$} & \multirow[b]{2}{*}{ Numerator } & \multirow[b]{2}{*}{ Denominator } \\
\hline & & Lower & Upper & & \\
\hline Sensitivity & 42.9 & 9.9 & 81.6 & 3 & 7 \\
\hline Specificity & 100 & 87.7 & 100 & 28 & 28 \\
\hline PPV & 100 & 29.2 & 100 & 3 & 3 \\
\hline PNV & 87.5 & 71.0 & 96.5 & 28 & 32 \\
\hline \multirow[t]{2}{*}{ Accuracy } & 88.6 & 73.3 & 96.8 & 31 & 35 \\
\hline & & \multicolumn{4}{|c|}{ Pathology Multigland } \\
\hline \multicolumn{2}{|c|}{ CT Multigland } & \multicolumn{2}{|c|}{ No } & Yes & Total \\
\hline \multicolumn{2}{|c|}{ No } & \multicolumn{2}{|c|}{28} & 4 & 32 \\
\hline \multirow{2}{*}{\multicolumn{2}{|c|}{ Yes }} & \multicolumn{2}{|c|}{0} & 3 & 3 \\
\hline & Total & \multicolumn{2}{|c|}{28} & 7 & 35 \\
\hline
\end{tabular}

disease. As before, the specificity of 4D-CT in ruling out multigland disease in this population was perfect (specificity = $100 \%, 95 \%$ CI, $87.2 \%, 100 \%$ ). Table 5 displays the diagnostic accuracy of $4 \mathrm{D}-\mathrm{CT}$ in detecting multigland disease, including those patients called negative by $4 \mathrm{D}-\mathrm{CT}$.

\section{Discussion}

4D-CT demonstrated high accuracy for the determination of both lesion side and quadrant location for single-gland disease. Our series suggests that 4D-CT agrees with the pathology findings in most of the cases and is a reliable choice for parathyroid adenoma detection. Importantly, 4D-CT accurately lateralized single-gland adenomas in $93 \%$ of cases, allowing the surgeon to perform a unilateral neck dissection with associated decreased morbidity and length of hospital stay. The ability to accurately lateralize the side of disease is a commonly used end point in parathyroid imaging because it enables a directed surgical approach. By comparison, sonography has a cited sensitivity for lateralization ranging from $57 \%$ to $86 \% .^{5,11}$

4D-CT appears to hold potential for detection of multi- 
gland disease. Although this technique demonstrated suboptimal sensitivity of $44 \%$ in patients with multigland disease, it was $100 \%$ specific for ruling out multigland disease, as no cases that were thought to have multigland disease by $4 \mathrm{D}-\mathrm{CT}$ had single-gland adenomas at pathology. These results compare favorably to those reported for sonography and sestamibi techniques. A review by Sugg et al, ${ }^{12}$ including 23 patients with multigland disease, found that the combination of sonography and sestamibi imaging correctly predicted multigland disease in $30 \%$ of patients, incorrectly called single-gland disease in $30 \%$, did not identify abnormal parathyroid glands in $30 \%$, and yielded discordant results in 10\%. In our cohort, 2 of the 3 false-negatives for parathyroid lesion detection were patients with multigland disease. Multigland lesions are particularly challenging to detect prospectively, likely related to the small lesion size. We would further posit, as other authors have suggested, that in the case of a negative $4 \mathrm{D}$-CT examination, there is presumed multigland disease. Importantly, some authors suggest a nonselective operative approach in cases of negative or discordant imaging studies due to the increased likelihood of multigland pathology. ${ }^{2,13}$

Unique angiographic and perfusion characteristics of parathyroid adenomas were described by Doppman and others in the 1970s, and used to identify abnormal hypervascular glands with preoperative digital subtraction angiography. ${ }^{9,14}$ A characteristic "blush" has been described following the injection of intra-arterial contrast, indicating the presence of an abnormal gland that is hypervascular compared with normal thyroid and parathyroid tissue. Similar principles are applied to contrast-enhanced CT, where hyperenhancement, location, and size are important discriminators in detecting parathyroid adenomas. ${ }^{4}$ DD-CT appears to further enhance detection, with the advantages of noncontrast imaging to distinguish the gland from surrounding thyroid tissue, early postcontrast imaging to assess for a hypervascular gland, and delayed postcontrast imaging to detect regions of altered enhancement and abnormal contrast retention.

It is important to be cognizant of the radiation dose delivered with CT, particularly multiphase CT. We use dose reduction techniques with $4 \mathrm{D}-\mathrm{CT}$, as described by Welling et al, ${ }^{8}$ including automatic tube current modulation and limiting the superior field of view to the mandibular teeth. Despite these techniques, 4D-CT delivers a significant radiation dose. Calculated $\mathrm{CTDI}_{\mathrm{vol}}$ measurements range from $19.8 \mathrm{mGy}$ (at $150 \mathrm{~mA}$ ) to $39.7 \mathrm{mGy}$ (at $300 \mathrm{~mA}$ ) per phase of imaging. These parameters are comparable with recently reported $\mathrm{CTDI}_{\mathrm{vol}}$ values for neck CT ranging from 23.0 to $34.7 \mathrm{mGy} .{ }^{15}$ We have chosen not to exclude the noncontrast examination, as we think it provides important diagnostic information, particularly in its ability to show attenuation differences in lesions contiguous with the thyroid gland. The risk-benefit discussion must weigh the cost, morbidity, and risk of formal neck exploration with the exposure from 4D-CT. The examination will likely only require performance on a single occasion.

There are several limitations to our study. First, this study is a retrospective review and was not designed to make a direct prospective comparison of the accuracy of sonography, sestamibi, and 4D-CT imaging for the detection of parathyroid pathology. Second, there is a selection bias for cases of ectopic and multigland disease because these are more difficult to di- agnose on traditional imaging modalities and thus more likely to progress to $4 \mathrm{D}$-CT imaging. Our patient population may be skewed by this factor, with a relatively higher proportion of these subtypes of adenomas. Third, although the technique of $4 \mathrm{D}-\mathrm{CT}$ at our institution has been performed with high accuracy, variability in diagnostic accuracy may occur across institutions, secondary to interpreter experience and study technique.

Nonetheless, our series demonstrates that 4D-CT is a valuable tool for appropriate operative planning. 4D-CT also shows a higher sensitivity and accuracy than commonly cited for sonography or sestamibi imaging. We believe the accuracy of this technique is particularly impressive when considering the patients referred for $4 \mathrm{D}-\mathrm{CT}$ frequently had equivocal or indeterminate findings on prior sonography and sestamibiSPECT studies. The multiplanar reconstruction capabilities provide beneficial spatial information for optimal operative planning and intervention.

\section{Conclusions}

4D-CT demonstrated a high diagnostic accuracy for singlegland disease in our cohort and high specificity in multigland disease. Importantly, 4D-CT predicted the correct side of single-gland adenomas in $93 \%$ of cases, allowing the surgeon to utilize a directed operative approach. Potential difficulties with the technique include radiation exposure and necessity of iodinated contrast media.

Disclosures: C. Douglas Phillips_Consultant: MedSolutions, Details: Consultant, stock options; Other Financial Relationships: Amirsys, Details: Write chapters.

\section{References}

1. Sosa JA, Udelsman R. Minimally invasive parathyroidectomy. Surg Oncol 2003;12:125-34

2. Johnson NA, Tublin ME, Ogilvie JB. Parathyroid imaging: technique and role in the preoperative evaluation of primary hyperparathyroidism. $A J R A m J$ Roentgenol 2007;188:1706-15

3. Arici C, Cheah WK, Ituarte PH, et al. Can localization studies be used to direct focused parathyroid operations? Surgery 2001;129:720-29

4. Randall GJ, Zald PB, Cohen JI, et al. Contrast-enhanced MDCT characteristics of parathyroid adenomas. AJR Am J Roentgenol 2009;193:W139-43

5. Rodgers SE, Hunter GJ, Hamberg LM, et al. Improved preoperative planning for directed parathyroidectomy with 4-dimensional computed tomography. Surgery 2006;140:932-40, discussion 940-41

6. Philip M, Guerrero MA, Evans DB, et al. Efficacy of 4D-CT preoperative localization in 2 patients with MEN 2A. J Surg Educ 2008;65:182-85

7. Mortenson MM, Evans DB, Lee JE, et al. Parathyroid exploration in the reoperative neck: improved preoperative localization with 4D-computed tomography. J Am Coll Surg 2008;206:888-95, discussion 895-96

8. Welling RD, Olson JA Jr, Kranz PG, et al. Bilateral retropharyngeal parathyroid hyperplasia detected with $4 \mathrm{D}$ multidetector row CT. AJNR Am J Neuroradiol 2010;32:E80-82

9. Miller DL, Doppman JL. Parathyroid angiography. Ann Intern Med 1987;107:942-43

10. Udelsman R, Aruny JE, Donovan PI, et al. Rapid parathyroid hormone analysis during venous localization. Ann Surg 2003;237:714-21

11. Siperstein A, Berber E, Mackey R, et al. Prospective evaluation of sestamibi scan, ultrasonography, and rapid PTH to predict the success of limited exploration for sporadic primary hyperparathyroidism. Surgery 2004;136:872-80

12. Sugg SL, Krzywda EA, Demeure MJ, et al. Detection of multiple gland primary hyperparathyroidism in the era of minimally invasive parathyroidectomy. Surgery 2004;136:1303-09

13. Chan RK, Ruan DT, Gawande AA, et al. Surgery for hyperparathyroidism in image-negative patients. Arch Surg 2008;143:335-37

14. Doppman JL. Parathyroid localization: arteriography and venous sampling. Radiol Clin North Am 1976;14:163-88

15. Russell MT, Fink JR, Rebeles F, et al. Balancing radiation dose and image quality: clinical applications of neck volume CT. AJNR Am J Neuroradiol 2008; 29:727-31. 